\title{
A THREE-DECADE OUTBURST OF THE LMC LUMINOUS BLUE VARIABLE R127 DRAWS TO A CLOSE
}

\author{
Nolan R. Walborn, ${ }^{1}$ Otmar Stahl, ${ }^{2}$ Roberto C. Gamen, ${ }^{3}$ Thomas Szeifert, ${ }^{4}$ Nidia I. Morrell, ${ }^{5}$ Nathan Smith, \\ Ian D. Howarth, ${ }^{7}$ Roberta M. Humphreys, ${ }^{8}$ Howard E. Bond, ${ }^{1}$ And Daniel J. Lennon ${ }^{1}$ \\ Received 2008 May 23; accepted 2008 July 3; published 2008 July 21
}

\begin{abstract}
The paradigmatic luminous blue variable R127 in the Large Magellanic Cloud has been found in the intermediate, peculiar early-B state, and substantially fainter in visual light, signaling the final decline from its major outburst that began between 1978 and 1980. This transformation was detected in 2008 January, but archival data show that it began between early 2005 and early 2007. In fact, significant changes from the maximum, peculiar A-type spectrum, which was maintained from 1986 through 1998, had already begun the following year, coinciding with a steep drop in visual light. We show detailed correspondences between the spectrum and light, in which the decline mimics the rise. Moreover, these trends are not monotonic but are characterized by multiple spikes and dips, which may provide constraints on the unknown outburst mechanism. Intensive photometric and spectroscopic monitoring of R127 should now resume, to follow the decline presumably back to the quiescent Ofpe/ WN9 state, in order to fully document the remainder of this unique observational opportunity.
\end{abstract}

Subject headings: Magellanic Clouds — stars: early-type — stars: individual (R127, HDE 269858) — stars: variables: other — stars: winds, outflows

\section{INTRODUCTION}

The luminous blue variable (LBV) phase is believed to represent a critical transition in the late evolution of all stars with initial masses greater than about $50 M_{\odot}$, during which they lose sufficiently large amounts of mass in recurrent explosive events to subsequently become classical WR stars. Although the physical mechanism producing these outbursts remains to be definitively identified, it is likely related to the Eddington limit (Humphreys \& Davidson 1994; Smith \& Owocki 2006), and there is ample phenomenological evidence to support the opening statement above. A fundamental observational characteristic of massive stellar populations is that the most luminous blue stars have no red counterparts. The LBVs lie just to the left of the inclined Humphreys-Davidson (HD; 1979, 1984, 1994; Humphreys 1983) limit in the H-R diagram, beyond which no stars are observed; that is, they appear to define the locus of an instability that prevents further redward evolution. During their outbursts, LBVs expand and develop denser, optically thick winds that reprocess the photospheric radiation to lower effective temperatures, such that they exhibit horizontal excursions across the HD limit, with increasingly cooler spectra and brighter visual magnitudes while maintaining constant bolometric luminosities. Following an outburst, they invert these displacements and return to the hotter, minimum spectrum with fainter visual magnitude. Most LBVs and numerous related objects now interpreted as quiescent LBVs have

\footnotetext{
${ }^{1}$ Space Telescope Science Institute, 3700 San Martin Drive, Baltimore, MD 21218; walborn@stsci.edu, bond@stsci.edu, lennon@stsci.edu. STScI is operated by AURA, Inc., under NASA contract NAS 5-26555.

${ }^{2}$ ZAH, Landessternwarte Königstuhl, 69117 Heidelberg, Germany; O.Stahl@1sw.uni-heidelberg.de.

${ }^{3}$ Complejo Astronómico El Leoncito, Avenida España 1412 Sur, J5402DSP, San Juan, Argentina. Member of Carrera del Investigador, CONICET, Argentina; rgamen@ casleo.gov.ar.

${ }^{4}$ European Southern Observatory, Alonso de Córdova 3107, Santiago, Chile; tszeifer@eso.org.

${ }^{5}$ Las Campanas Observatory, Carnegie Observatories, Casilla 601, La Serena, Chile; nmorrell@1co.cl.

${ }^{6}$ Astronomy Department, University of California, 601 Campbell Hall, Berkeley, CA 94720; nathans@astro.berkeley.edu.

${ }^{7}$ Department of Physics and Astronomy, University College London, Gower Street, London WC1E 6BT, UK; idh@star.ucl.ac.uk.

${ }^{8}$ Astronomy Department, 116 Church Street, SE, University of Minnesota, Minneapolis, MN 55455; roberta@isis.spa.umn.edu.
}

nitrogen-rich circumstellar nebulae, produced by prior outbursts thousands of years earlier (Walborn 1982; Stahl \& Wolf 1986b; Stahl 1987; Nota et al. 1992, 1995, 1996; Clampin et al. 1993; Davidson et al. 1993; Smith et al. 1994, 1997, 1998; Pasquali et al. 1999; Lamers et al. 2001).

$\eta$ Carinae is the most famous and extreme LBV (Davidson \& Humphreys 1997), but it is located in a giant H II region associated with some of the most massive stars known, well above $100 M_{\odot}$ (Walborn et al. 2002). Given the uncertainty about the LBV mechanism, it is likewise unclear whether or not $\eta$ Car represents a more spectacular version of typical LBVs, which are located in more evolved regions and have initial masses between 50 and $100 M_{\odot}$. Well observed examples of the latter are AG Carinae (Wolf \& Stahl 1982; Stahl 1986; Hutsemékers \& Kohoutek 1988; Walborn \& Fitzpatrick 2000; Stahl et al. 2001); and R71 (Wolf et al. 1981) and S Doradus (Massey 2000 and references therein) in the Large Magellanic Cloud (LMC). The known incidence of these objects in other Local Group galaxies has been greatly extended by Massey et al. (2007).

Radcliffe (R)127 (Feast et al. 1960) = HDE 269858 $=$ Sanduleak (1970; Sk) -69 220 is arguably the most important typical LBV. In fact, taking into account this report, it is not an overstatement that it is comparable to $\eta$ Car and even SN 1987A, with regard to what it may teach us about late massive stellar evolution. Its highly peculiar spectrum was classified as O Iafpe by Walborn (1977; observation date 1975 December), later reformulated to Ofpe/WN9 by Walborn (1982; echelle observation 1978 November). A number of similar objects were found in the LMC (Bohannan \& Walborn 1989), which have subsequently been reclassified as WN9-11 types by Crowther \& Smith (1997). In addition to the normal Of selective emission lines of He II $\lambda 4686$ and $\mathrm{N}$ III $\lambda \lambda 4634-4640-4642$, these

\footnotetext{
${ }^{9}$ Feast et al. denoted it as HDE $269858 \mathrm{f}$ for "following" because of the companion $3^{\prime \prime}$ (north)west, now measured at $y=12.12$ and classified as B0 Ia (N weak) by Heydari-Malayeri et al. (2003). It is important to note that the HDE-based photographic magnitude of 11.4 given by Feast et al. must refer to the combined light of the pair, whereas their description of it as a "double" likely implies comparable magnitudes, consistently with the foregoing values. Most or all of the photometry discussed in this Letter includes both stars; accordingly, all values presented have been corrected for the companion, which is significant at the fainter LBV magnitudes.
} 


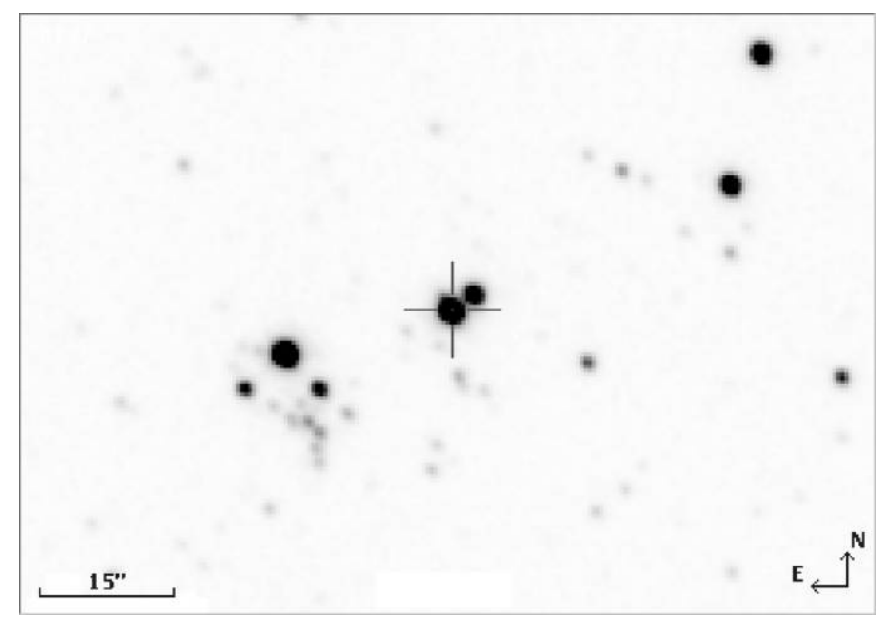

Fig. 1.-LCO Swope $1 \mathrm{~m}, 3 \mathrm{~s} V$ exposure of R127 (marked with a cross) and NGC 2055, obtained on 2008 March 19. The seeing was 1.5". Compare with the image taken at R127 maximum light, shown by Walborn et al. (1991). (Note that the scale given in that caption is incorrect.)

spectra display other emission features and $\mathrm{P}$ Cygni profiles including Balmer and $\mathrm{He} \mathrm{I}$ lines, together with very narrow $\mathrm{N}$ III and Si IV absorptions indicative of unusually extended atmospheres. In 1982, R127 was discovered to have entered a classical LBV outburst (Stahl et al. 1983; Walborn 1983), thus establishing the Ofpe/WN9 category as a quiescent LBV state. Several other members of the category were subsequently discovered to possess LBV circumstellar nebulae (references above), supporting the foregoing generalization. R127 is located in a compact subgroup of a small, evolved cluster (NGC 2055) containing several mid-O through early-B supergiants and devoid of nebulosity (Walborn et al. 1991; Heydari-Malayeri et al. 2003), south of 30 Doradus and not far from SN 1987A. This Letter reports that R127 has likely now begun its final decline from the current outburst; it is thus the largest and most extensive LBV event that will have been covered from start to finish with modern instrumentation.

\section{OBSERVATIONS}

The discovery of R127's decline began with inquiries by N. $\mathrm{R}$. W. of several specialists at and immediately following IAU Symposium 250 in 2007 December, regarding the current status of its monitoring. When no such program could be identified, check observations were requested of and performed by R. C. G. at the CASLEO Observatory in Argentina, and by N. I. M. at the Las Campanas Observatory (LCO) in Chile. These data immediately revealed that R127 was then in an intermediate, peculiar B-type state with strong He I P Cygni profiles, as well as N II, Si III, and Si IV features (Walborn et al. 2008), completely different from the maximum, peculiar A-type spectrum dominated by Fe II and Ti II, in which it had been in 2002 when intensive monitoring by O. S. and T. S. ceased. Direct images from the LCO $1 \mathrm{~m}$ Swope telescope (Fig. 1) requested by N. I. M. and measured by R. C. G. showed a significant decline in visual light with respect to the previous AAVSO data. Subsequent archival searches and a casual comment by N. R. W. to N. S. yielded, respectively, the critical spectroscopic observations of 2007 February from the European Southern Observatory (PI B. Davies), which shows that R127 was already in the early-B state then; and of the unique transitional spectrum in 2005 February from LCO. All of the high-resolution spectroscopic observations discussed here are specified in Table 1. Key segments of them are reproduced in Figures 2-4. A summary light curve
TABLE 1

OBSERVATIONS

\begin{tabular}{lcccc}
\hline \hline \multicolumn{1}{c}{ Date } & Telescope & Instr. & $R$ & PI/Observer \\
\hline 1984 Aug 31 & ESO $3.6 \mathrm{~m}$ & CASPEC & 20,000 & B. Wolf \\
1986 Aug 23 & ESO $3.6 \mathrm{~m}$ & CASPEC & 20,000 & B. Wolf \\
1987 Nov 6 & ESO $3.6 \mathrm{~m}$ & CASPEC & 20,000 & B. Wolf \\
1989 Jan 22 & ESO $3.6 \mathrm{~m}$ & CASPEC & 20,000 & O. S. \\
1989 Dec 16 & ESO $3.6 \mathrm{~m}$ & CASPEC & 20,000 & B. Wolf \\
1991 Jan 28 & ESO $3.6 \mathrm{~m}$ & CASPEC & 20,000 & B. Wolf \\
1991 Dec 16 & ESO $3.6 \mathrm{~m}$ & CASPEC & 20,000 & B. Wolf \\
1993 Dec 23 & ESO $3.6 \mathrm{~m}$ & CASPEC & 20,000 & B. Wolf \\
1995 Feb 16 & ESO $3.6 \mathrm{~m}$ & CASPEC & 20,000 & B. Wolf \\
1996 Jan 26 & ESO $3.6 \mathrm{~m}$ & CASPEC & 20,000 & B. Wolf \\
1997 Jan 26 & ESO $3.6 \mathrm{~m}$ & CASPEC & 20,000 & B. Wolf \\
1998 Oct 11 & ESO $1.5 \mathrm{~m}$ & FEROS & 48,000 & O. S. \\
1999 Jul 26 & ESO $1.5 \mathrm{~m}$ & FEROS & 48,000 & O. S. \\
2002 Sep 18 & ESO $8.0 \mathrm{~m}$ & UVES $^{\mathrm{b}}$ & 80,000 & T. S. \\
2005 Feb 21 & LCO $6.5 \mathrm{~m}$ & MIKE $^{\mathrm{c}}$ & 60,000 & N. S. \\
2007 Feb 20 & ESO $2.2 \mathrm{~m}$ & FEROS $^{\mathrm{a}}$ & 48,000 & B. Davies \\
2008 Jan 13 & CASLEO $2.2 \mathrm{~m}$ & REOSC $^{\mathrm{d}}$ & 25,000 & R. C. G. \\
2008 Feb 13 & CASLEO $2.2 \mathrm{~m}$ & REOSC & 25,000 & R. C. G. \\
2008 May 14 & ESO $2.2 \mathrm{~m}$ & FEROS $^{4}$ & 48,000 & N. I. M. \\
\hline
\end{tabular}

${ }^{a}$ Fiber-fed Extended Range Optical Spectrograph.

${ }^{\mathrm{b}}$ Ultraviolet-Visual Echelle Spectrograph.

${ }^{c}$ Magellan Inamori Kyocera Echelle.

${ }^{\mathrm{d}}$ Echelle spectrograph on loan from the Institut d'Astrophysique de Liège.

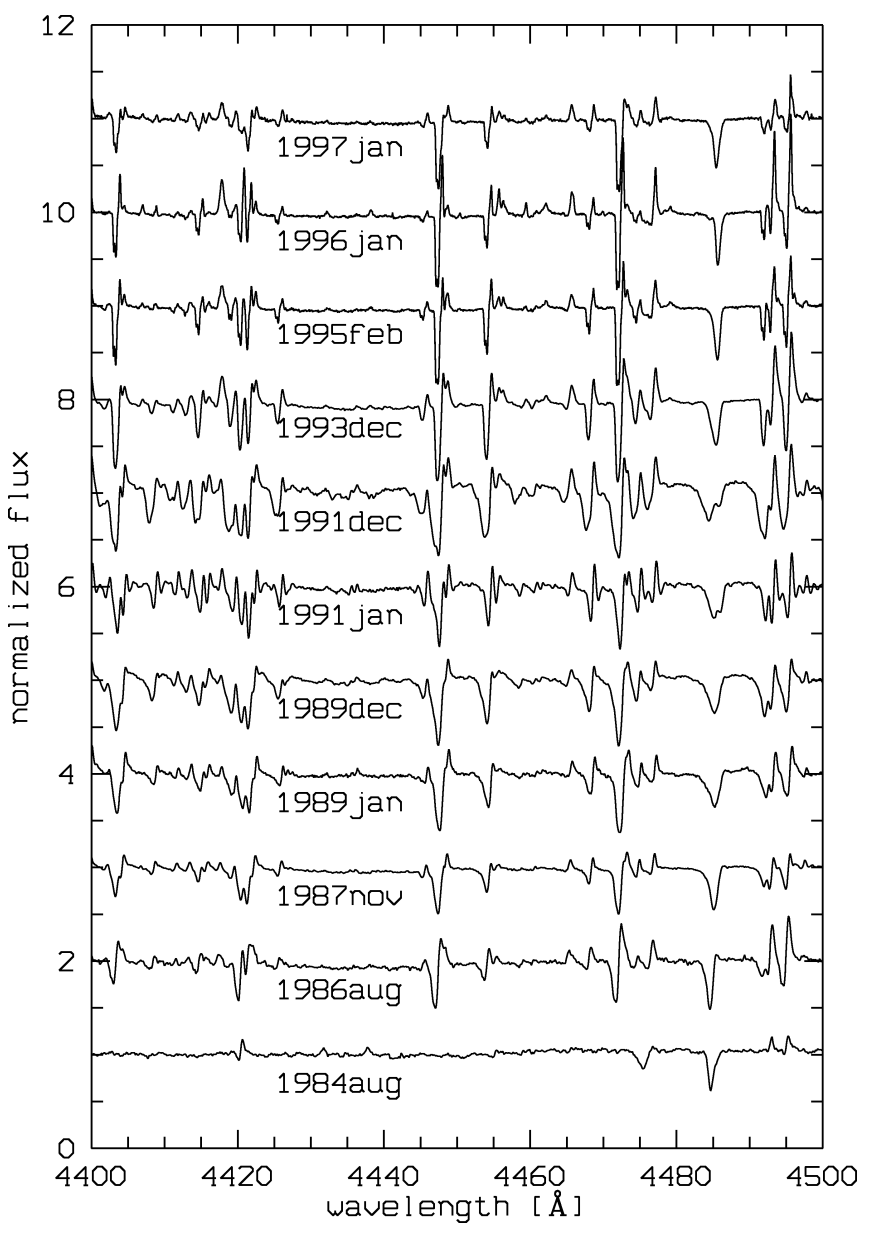

FIG. 2.-Time sequence of rectified spectroscopic observations of R127 (see Table 1). The wavelength scale in the present spectroscopic illustrations is heliocentric; i.e., R127's radial velocity of $293 \mathrm{~km} \mathrm{~s}^{-1}$ or $4.4 \AA$ at $4500 \AA$ is included. The 1984 August observation shows strong He I $\lambda 4471$ and $\mathrm{Mg}$ II $\lambda 4481$ absorption lines; the latter is present throughout and is double in 1991 (see text). The strongest P Cygni profiles are due to Fe II $\lambda \lambda 4414-17,4489$, 4491 and Ti II $\lambda \lambda 4444,4468$. 


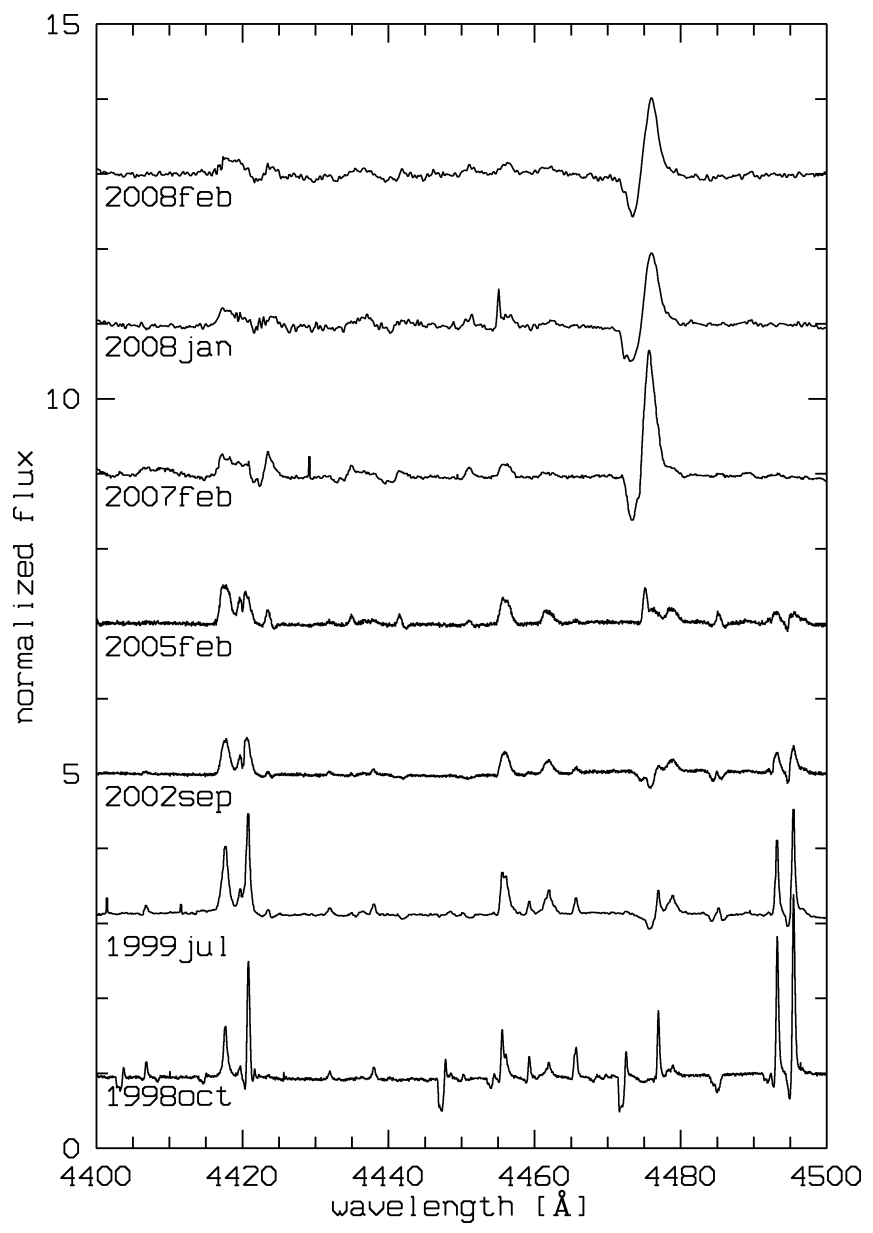

Fig. 3.-Continuation of the Fig. 2 time sequence, but with higher resolutions (Table 1). In 1998 the Ti II (IP $13.6 \mathrm{eV}$ ) features weakened substantially and they disappeared entirely in 1999 , while Fe II $(16.2 \mathrm{eV})$ remained strong. The behavior of the 4414-17 $\AA$ complex with time is remarkable and is caused by the increasing contribution of [Fe II] relative to $\mathrm{Fe}$ II at nearly the same wavelengths; the former arises farther out in the wind where the velocity is higher. Note also the transition from Fe II $\lambda 4473$ to He I $\lambda 4471$ from 1999 through 2005, and the double Mg II $\lambda 4481$ absorption in 2002. The 20072008 spectra are dominated by the very strong He I $\lambda 4471$ P Cygni profile.

is presented in Figure 5, extending from 1982 through early 2008. The 2002 and earlier data are representative of many more obtained by O. S. and T. S., whereas the 2005-2008 are all the high-resolution, blue-violet observations of which we are aware. Here we shall describe the major spectral transformations with reference to the light curve.

\section{DISCUSSION}

The R127 outburst through 1986 was extensively documented by Stahl et al. (1983), Stahl \& Wolf (1986a), and Wolf et al. (1988). As they discuss, the spectroscopic observation by Walborn (1982) and subsequent photometry limit the onset of the outburst to the interval 1978-1980. Most of the spectroscopic data in the early papers were photographic; only the digital data are now available in electronic form and are shown here; thus it is essential to refer to the figures in those papers for the complete early record, and also for detailed line identifications in the A-type spectra.

The early outburst photometry twice appeared to show declines from maxima, in 1982 and 1984, but these are now seen to be only inflections during the rise to the true maximum (Fig. 5), when R127 became the brightest star in the LMC. The first outburst

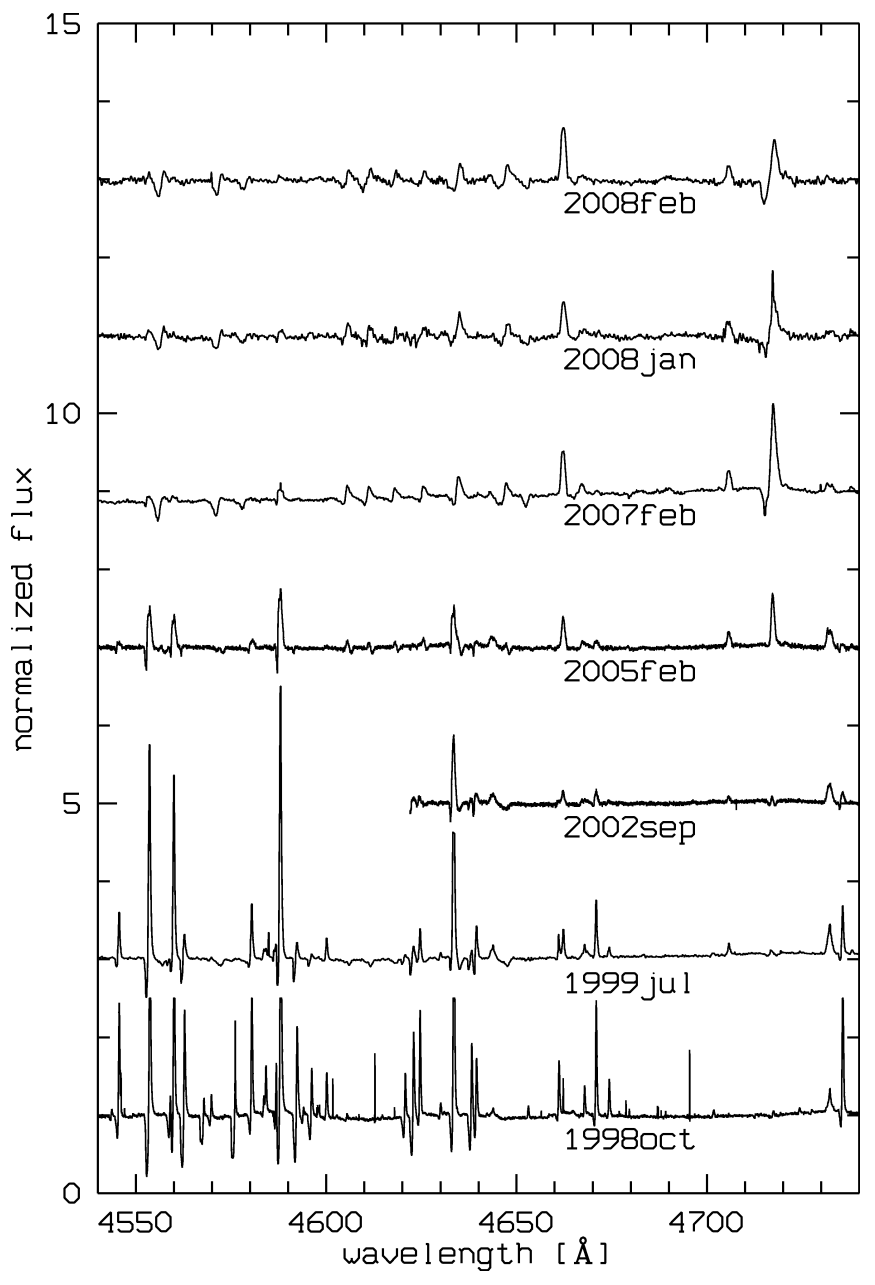

FIG. 4.- Same spectrograms as in Fig. 3, at somewhat longer wavelengths. The four strongest emission features in 1998 October reach about 6 continuum units and have been truncated here, as has the last in 1999 July; they are Fe II $\lambda \lambda 4549$, $4556,4584,4629$. In addition to the P Cygni profile of He I $\lambda 4713$, the B-type features Si III $\lambda \lambda 4552,4568,4575$ and $N$ II $\lambda \lambda 4601,4607,4614,4621,4631,4643$ are prominent in the 2007-2008 spectra, as are [Fe III] $\lambda \lambda 4658,4701.5$.

spectroscopic observation, in 1982 January, showed a peculiar early B-type spectrum, similar but not identical to the current state (Stahl et al. 1983). Subsequently (Stahl \& Wolf 1986a), the spectrum changed to $\sim \mathrm{B} 7$ (based on near equality of $\mathrm{He} \mathrm{I} \lambda 4471$ and Mg II $\lambda 4481$; Walborn \& Fitzpatrick 1990) in 1983 February during the decline from the 1982 light curve peak; then to $\sim \mathrm{B} 9$

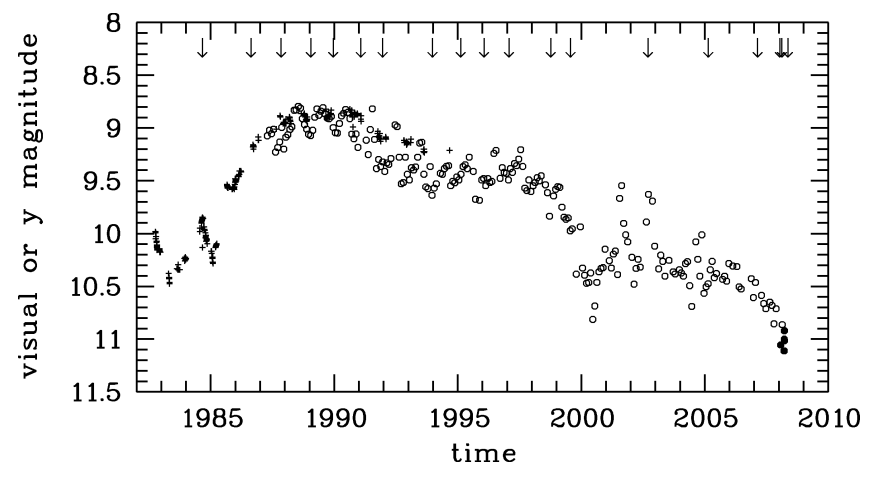

FIG. 5.-Composite visual light curve of R127 from 1982 through early 2008. All measurements have been corrected for the companion. Crosses: $y$ data from Sterken et al. (1995). Open circles: Monthly averages of AAVSO visual observations. Filled squares: New LCO $V$ data. The epochs of the spectroscopic observations discussed here are marked by arrows at the top. 
(shown in Fig. 2) coinciding with the peak of 1984 August; and back again to $\sim$ B7 in 1985 January during the decline from the 1984 peak. Then, as the visual light continued to increase, the full A-type spectrum developed in 1986 (Wolf et al. 1988) and was maintained through 1998 (Figs. 2-3).

These relationships between the spectrum and light curve encourage a detailed comparison between the spectral types and apparent magnitudes, which is given in Table 2. It is seen that the correlation is indeed very well defined, and that it applies equally to both the rise and decline, insofar as they have been covered. This correlation is, of course, a result of the "constant" bolometric luminosity during the outburst, as shown in the table. The scatter in the derived bolometric magnitudes is not surprising, given the different sources of the corrections and the fact that they are computed for normal supergiants, as well as the uncertainties in the classification of these peculiar spectra.

Even more detailed effects occur, e.g., the apparent doubling of the Mg II line during 1991 (Fig. 2), when the small decline from the maximum hump (with an upward spike?) to an extended plateau took place (Fig. 5). It is quite possible that other small differences among the spectra are related to spectroscopically unresolved fine structure in the light curve. A larger spectral change is evidently related to the steep decline in light beginning in 1999 (Figs. 3-4). There is extensive spectroscopic coverage during 2002, not shown here, which may elucidate the strong spike in light during that year; indeed, the Mg II line again doubled during September, near the light curve peak. The 2002 observation shown might again be classified as $\sim \mathrm{B} 7$, from the $\mathrm{He} \mathrm{I} / \mathrm{Mg}$ II absorption ratio, albeit with residual A-type emission lines.

A striking feature of Figure 3 is the apparent monotonic broadening of the A-type lines as they fade, which is caused by the gradual dominance of [Fe II] farther out in the wind over Fe II at nearly the same wavelengths, as discussed by Stahl et al. (2001) in AG Car. In contrast, the He I $\lambda 4471$ emission line was very narrow when it first appeared in 2005. The recent spectra also display changing structure in the Balmer P Cygni absorption components, not shown here, analogous to what was observed during the R127 rise and in AG Car. All of these features in R127 will be measured and discussed in subsequent publications.

It is now vital to increase the frequency of observations during the coming years, to document R127's final decline to the minimum, quiescent state more thoroughly. In particular, it
TABLE 2

Spectral Types and Apparent Magnitudes

\begin{tabular}{ccccc}
\hline \hline $\begin{array}{c}\text { Visual } \\
\text { Magnitude }\end{array}$ & $\begin{array}{c}\text { Spectral } \\
\text { Type }\end{array}$ & Epochs & BC & $m_{\text {bol }}{ }^{\mathrm{e}}$ \\
\hline $11.8-12.2$ & Ofpe & $\leq 1978$ & $-2.8^{\mathrm{a}}$ & $9.0-9.4$ \\
$10.6-11.0$ & B1-2 & 1982 Jan, 2007 Feb, 2008 Jan-Feb & $-1.9^{\mathrm{b}}$ & $8.7-9.1$ \\
$10.2-10.3$ & B7 & 1983 Feb, 1985 Jan, 2002 Sep? & $-0.6^{\mathrm{c}}$ & $9.6-9.7$ \\
9.8 & B9 & 1984 Aug & $-0.4^{\mathrm{c}}$ & 9.4 \\
$8.8-9.5$ & A & 1986 Mar-1998 Oct & $-0.1^{\mathrm{d}}$ & $8.7-9.4$ \\
\hline
\end{tabular}

${ }^{a}$ Martins et al. (2005) (O9 I).

${ }^{\mathrm{b}}$ Lanz \& Hubeny (2007) (20,000 K, lowest $g$ ).

Humphreys \& McElroy (1984) (supergiants).

${ }^{\mathrm{d}}$ Schiller \& Przybilla (2008) (Deneb, A2 Ia).

${ }^{\mathrm{e}}$ With $E_{B-V}=0.20$ and $V_{0}-M_{V}=18.6, m_{\mathrm{bol}}=9.0$ corresponds to $M_{\mathrm{bol}}=-10.2$.

will be of some interest to catch the reappearance of the He II and $\mathrm{N}$ III Of emission lines-provided that an infrared catastrophe as in $\eta$ Car does not obscure the star entirely. It is possible that the high-ionization lines may already be very weakly present in the 2008 February and May observations.

We thank the Carnegie Supernova Project team, in particular M. Phillips, W. Krzeminski, Sergio González, and Carlos Contreras, for the LCO Swope photometric images. This Letter includes an observation from a $6.5 \mathrm{~m}$ Magellan Telescope at LCO, Chile. The Letter is in large part based on observations collected by multiple programs at both the La Silla and Paranal sites of the European Organization for Astronomical Research in the Southern Hemisphere, Chile. The Complejo Astronómico El Leoncito (CASLEO) in San Juan is operated under agreement between CONICET, SeCyT, and the Universities of La Plata, Córdoba, and San Juan, Argentina. The CCD and data-acquisition system used there are supported under US NSF grant AST 90-15827 to R. M. Rich. We acknowledge with thanks the variable star observations from the AAVSO International Database contributed by observers worldwide and used in this research. Publication support was provided by NASA through grant GO-11212.03-A from STScI, which is operated by AURA, Inc., under NASA contract NAS 5-26555. Finally, we thank the anonymous referee for requesting that the effects of the companion on the LBV photometry be investigated, which improved the accuracy of the presentation.

\section{REFERENCES}

Bohannan, B., \& Walborn, N. R. 1989, PASP, 101, 520

Clampin, M., et al. 1993, ApJ, 410, L35

Crowther, P. A., \& Smith, L. J. 1997, A\&A, 320, 500

Davidson, K., \& Humphreys, R. M. 1997, ARA\&A, 35, 1

Davidson, K., et al. 1993, ApJ, 411, 336

Feast, M. W., Thackeray, A. D., \& Wesselink, A. J. 1960, MNRAS, 121, 337

Heydari-Malayeri, M., Meynadier, F., \& Walborn, N. R. 2003, A\&A, 400, 923

Humphreys, R. M. 1983, ApJ, 269, 335

Humphreys, R. M., \& Davidson, K. 1979, ApJ, 232, 409 . 1984, Science, 223, 243

1994, PASP, 106, 1025

Humphreys, R. M., \& McElroy, D. B. 1984, ApJ, 284, 565

Hutsemékers, D., \& Kohoutek, L. 1988, A\&AS, 73, 217

Lamers, H. J. G. L. M., et al. 2001, ApJ, 551, 764

Lanz, Th., \& Hubeny, I. 2007, ApJS, 169, 83

Martins, F., Schaerer, D., \& Hillier, D. J. 2005, A\&A, 436, 1049

Massey, P. 2000, PASP, 112, 144

Massey, P., et al. 2007, AJ, 134, 2474

Nota, A., Livio, M., Clampin, M., \& Schulte-Ladbeck, R. 1995, ApJ, 448, 788

Nota, A., et al. 1992, ApJ, 398, 621 1996, ApJS, 102, 383

Pasquali, A., Nota, A., \& Clampin, M. 1999, A\&A, 343, 536

Sanduleak, N. 1970, Contrib. CTIO 89

Schiller, F., \& Przybilla, N. 2008, A\&A, 479, 849
Smith, L. J., Crowther, P. A., \& Prinja, R. K. 1994, A\&A, 281, 833

Smith, N., \& Owocki, S. P. 2006, ApJ, 645, L45

Smith, L. J., et al. 1997, MNRAS, 290, 265 1998, ApJ, 503, 278

Stahl, O. 1986, A\&A, 164, 321 (erratum 170, 197) 1987, A\&A, 182, 229

Stahl, O., \& Wolf, B. 1986a, A\&A, 154, 243 1986b, A\&A, 158, 371

Stahl, O., et al. 1983, A\&A, 127, 49

- 2001, A\&A, 375, 54

Sterken, C., et al. 1995, A\&AS, 113, 31

Walborn, N. R. 1977, ApJ, 215, 53

1982, ApJ, 256, 452 1983, IAU Circ. 3767

Walborn, N. R., \& Fitzpatrick, E. L. 1990, PASP, 102, 379 2000, PASP, 112, 50

Walborn, N. R., et al. 1991, in IAU Symp. 143, Wolf-Rayet Stars and Interrelations with Other Massive Stars in Galaxies, ed. K. A. van der Hucht \& B. Hidayat (Dordrecht: Kluwer), 505 2002, AJ, 123, 2754 2008, IAU Circ. 8913

Wolf, B., Appenzeller, I., \& Stahl, O. 1981, A\&A, 103, 94

Wolf, B., \& Stahl, O. 1982, A\&A, 112, 111

Wolf, B., Stahl, O., Smolinski, J., \& Cassatella, A. 1988, A\&AS, 74, 239 